\title{
Oviposição dos ácaros predadores Agistemus floridanus Gonzalez, Euseius concordis (Chant) e Neoseiulus anonymus (Chant \& Baker) (Acari) em resposta a diferentes tipos de alimento
}

\author{
Noeli Juarez Ferla ${ }^{1} \&$ Gilberto José de Moraes ${ }^{2}$
}

\begin{abstract}
${ }^{1}$ Museu de Ciências Naturais, Centro Universitário UNIVATES. 95900-000 Lajeado, Rio Grande do Sul, Brasil. E-mail: njferla@fates.tche.br

2 Departamento de Entomologia, Fitopatologia e Zoologia Agrícola, Escola Superior de Agricultura "Luiz de Queiroz". 13418-900 Piracicaba, São Paulo, Brasil. Bolsista do CNPq. E-mail: gjmoraes@carpa.ciagri.usp.br
\end{abstract}

\begin{abstract}
Oviposition of the predators Agistemus floridanus Gonzalez, Euseius concordis (Chant) and Neoseiulus anonymus (Chant \& Baker) (Acari) in response the different kinds of food. Phytoseiidae and Sigmaeidae are the most common predatory mites on rubber tree leaves in the State of Mato Grosso, associated with phytophagous mites of the families Eriophyidae, Tenuipalpidae and Tetranychidae. The aim of this work was to compare the effect of different kinds of food, including different species of mites commonly found on the rubber tree in Mato Grosso, and one kind of pollen, on the oviposition of the predators Agistemus floridanus Gonzalez, 1965 (Stigmaeidae), Euseius concordis (Chant, 1959) and Neoseiulus anonymus (Chant \& Baker, 1965), both Phytoseiidae. Those predators are common on rubber tree leaves in Mato Grosso. For the tests with A. floridanus, discs of $2 \mathrm{~cm}$ in diameter of rubber tree leaves were used as substrate. Food provided to the predators were the mites Calacarus heveae Feres, 1992, Oligonychus gossypii (Zacher, 1921), Polyphagotarsonemus latus (Banks, 1904), Tenuipalpus heveae Baker, 1945 and Tetranychus mexicanus (McGregor, 1950), and pollen of Typha angustifolia L.. Agistemus floridanus accepted a larger variety of foodthan other predators. This was the only predator with high oviposition rate when fed $C$. heveae and $T$. heveae, the phytophagous mites considered most important on rubber trees. Euseius concordis and $A$. floridanus had nearly the same oviposition rates when fed pollen of $T$. angustifolia (ca. one egg per female per day). Neoseiulus anonymus had the highest oviposition rate than other predators when fed $O$. gossypii and $T$. mexicanus. Polyphagotarsonemus latus was the least suitable food for the predators studied.
\end{abstract}

KEY WORDS. Acari, biological control, fecundity, rubber tree.

As espécies mais comuns de ácaros fitófagos em folhas de seringueira (Hevea brasiliensis Muell. Arg.) no Estado do Mato Grosso pertencem às famílias Eriophyidae, Tarsonemidae, Tenuipalpidae e Tetranychidae (Ferla \& Moraes 2002). Devido às altas populações observadas, ao dano causado às folhas e à freqüência com que ocorrem em seringais do Mato Grosso, Calcarus heveae Feres, 1992 e Tenuipalpus heveae Baker, 1945 são considerados os ácaros fitófagos mais importantes nesta cultura (Feres 1992, 2000, FerLa \& Moraes 2002). De acordo com Vieira \& Gomes (1999), C. heveae pode provocar níveis de desfolha acima de 75\% em clones RRIM 600. Segundo Feres (2000), alguns produtores afirmam que $C$. heveae ocasiona perdas de até $30 \%$ na produção de látex de alguns clones de $H$. brasiliensis. Tenuipalpus heveae ataca principalmente a face abaxial dos folíolos (Feres 2000, FerLa \& Moraes 2002), causando o amarelecimento destes e aparentemente contribuindo para sua queda prematura. Além destas, outras espécies de ácaros fitófagos provocam danos a esta cultura. Polyphagotarsonemus latus (Banks, 1904) fica restrito às brotações, podendo provocar o aparecimento de folhas deformadas ou fendidas (FLeCHTMANN \& ARleu 1984). Oligonychus gossypii (Zacher, 1921) e Tetranychus mexicanus (McGregor, 1950) podem aparecer ocasionalmente em altos níveis na estação seca, após o enfolhamento das plantas e antes da intensificação das precipitações (FAzolin \& Pereira 1989, Feres 2000, Ferla \& Moraes 2002).

Ácaros Phytoseiidae e Stigmaeidae são os predadores mais comuns em folhas de seringueira no sudeste e centro-oeste do Brasil, sendo a maior diversidade apresentada pelos fitoseídeos (Feres 2000, Ferla \& Moraes 2002). Conhecer a interação de predadores e presas na cultura da seringueira é importante para subsidiar estudos populacionais de inimigos naturais e para o manejo de pragas nesta cultura. O objetivo deste trabalho foi dar um primeiro passo neste sentido, determinando a aceitação de ácaros fitófagos comuns em seringueira como fonte de ali- 
mento para Agistemus floridanus Gonzalez, 1965, Euseius concordis (Chant, 1959) e Neoseiulus anonymus (Chant \& Baker, 1965), em comparação com um tipo de pólen que em outros estudos tem se mostrado alimento favorável a vários predadores. A aceitação destes alimentos foi inferida a partir de uma análise dos níveis de oviposição destes predadores em condições de laboratório.

\section{MATERIAL E MÉTODOS}

Este trabalho foi realizado nos laboratórios da Escola Superior de Agricultura "Luiz de Quiroz" (ESALQ/USP), em Piracicaba, São Paulo, utilizando ácaros provenientes de colônias de laboratório iniciadas com espécimes coletados em folha de seringueira no Estado do Mato Grosso, há cerca de três meses. Euseius concordis e A. floridanus foram provenientes de folíolos de seringueira postos com a face adaxial para baixo, sobre um pedaço de espuma de náilon umedecida, em uma bandeja plástica parcialmente coberta com um pedaço de papelão para evitar a incidência de luz direta sobre as unidades. Estes ácaros foram alimentados com uma mistura de diferentes estádios de O. gossypii e T. heveae.

As colônias de E. concordis e N. anonymus foram mantidas em unidades de criação semelhantes às descritas por McMurTRY \& Scriven (1965). Cada unidade constou de uma placa de Paviflex $^{\grave{O}}$ colocada sobre um pedaço de papelão. Estes ácaros foram alimentados com pólen de Typha angustifolia L. e uma mistura de diferentes estádios de Tetranychus urticae Koch, 1836 sobre pedaços de folhas de feijão-de-porco (Canavalia ensiformis (L.) D.C.) infestadas. Os grãos de pólen foram colocados sobre lamínulas de microscopia e estas postas na unidade de criação. A cada dois dias, as lamínulas foram substituídas por novas lamínulas com pólen. Os diferentes estádios de T. urticae foram também oferecidos a cada dois dias.

O tipo de unidade experimental utilizada para cada espécie de predador foi aquela que em testes preliminares se mostraram mais adequadas para esta finalidade, tendo em vista o diferente comportamento de cada predador. Os tratamentos consistiram da alimentação de cada espécie de ácaro predador com uma mistura de diferentes estádios de T. mexicanus, $O$. gossypii, T. heveae, C. heveae e P. latus, ou com pólen de $T$. angustifolia.

As unidades de criação, assim como as unidades experimentais, foram mantidas em uma câmara climatizada a $25 \pm 1^{\circ} \mathrm{C}$, fotofase de 12 horas e umidade relativa de $80 \pm 5 \%$.

\section{Procedimentos experimentais com $A$. floridanus}

Discos de dois centímetros de diâmetro recortados de folíolos de seringueira foram colocados com a face adaxial para baixo dentro de placas de plástico $\left(\right.$ Magipack $\left.^{\circ}\right)$. Para cada tipo de alimento, foram utilizados 15 discos, cada um com uma fêmea. As fêmeas utilizadas nos testes foram retiradas ao acaso da criação estoque de predadores e mostravam-se aparentemente sadias. Estas foram transferidas para novas arenas a cada três dias. Fêmeas mortas até o quarto dia não foram consideradas nos cálculos.

\section{Procedimentos com E. concordis e N. anonymus}

Os testes com estes predadores foram feitos sobre folíolos de seringueira postos com a face adaxial para baixo sobre pedaços de esponja umedecida no interior de bandejas de alumínio. As margens dos folíolos foram recobertas com tiras de algodão hidrófilo também umedecido.

Para cada tipo de alimento, utilizaram-se quatro repetições em um delineamento experimental inteiramente casualizado. Cada parcela foi constituída por 10 fêmeas aparentemente sadias, tomadas ao acaso da unidade de criação. As fêmeas foram transferidas para novos substratos a cada três dias.

\section{Parâmetro avaliado e análise}

Cada teste teve duração de 11 dias, fazendo-se diariamente a contagem e a retirada dos ovos postos. Para a realização dos cálculos, descartou-se o primeiro dia de oviposição, devido à possível interferência da alimentação anterior do ácaro.

As médias de oviposição dos diferentes tratamentos foram comparadas pelo teste de Tukey, utilizando o programa estatístico SANEST, desenvolvido pelo Centro de Informática na Agricultura da ESALQ/USP.

\section{RESULTADOS}

A oviposição diária de $A$. floridanus foi igual ou superior a um ovo por fêmea quando estes ácaros foram alimentados com C. heveae, T. heveae, T. mexicanus, e pólen de T. angustifólia, diferindo significativamente da oviposição quando alimentando-se de O. gossypii e P. latus (Tab. I).

Tabela I. Oviposição média diária ( \pm erro padrão da média) de Agistemus floridanus, Euseius concordis e Neoseiulus anonymus em diferentes tipos de alimento, a $25 \pm 1{ }^{\circ} \mathrm{C}$, fotofase de 12 horas e umidade relativa de $80 \pm 5 \%$.

\begin{tabular}{lccc}
\hline \multicolumn{1}{c}{ Tipos de alimento } & A. floridanus & E. concordis & N. anonymus \\
\hline Calacarus heveae & $1,5 \pm 0,1 \mathrm{a}$ & $0,01 \pm 0,20 \mathrm{~b}$ & $0,01 \pm 0 \mathrm{c}$ \\
Oligonychus gossypii & $0,4 \pm 0,1 \mathrm{~b}$ & $0,09 \pm 0,01 \mathrm{~b}$ & $1,20 \pm 0,40 \mathrm{~b}$ \\
Polyphagotarsonemus latus & $0,3 \pm 0,2 \mathrm{~b}$ & $0,05 \pm 0,01 \mathrm{~b}$ & $0,40 \pm 0,10 \mathrm{c}$ \\
Tenuipalpus heveae & $1,7 \pm 0,2 \mathrm{a}$ & $0,10 \pm 0,20 \mathrm{~b}$ & $0 \mathrm{c}$ \\
Tetranychus mexicanus & $1,0 \pm 0,1 \mathrm{a}$ & $0,04 \pm 0,08 \mathrm{~b}$ & $2,00 \pm 0,10 \mathrm{a}$ \\
Pólen de Typha angustifolia & $1,0 \pm 0,1 \mathrm{a}$ & $0,90 \pm 0,20 \mathrm{a}$ & $0,01 \pm 3,10 \mathrm{c}$ \\
\hline
\end{tabular}

Euseius concordis teve oviposição diária de cerca de um ovo por fêmea quando alimentado com pólen de T. angustifolia, enquanto que com os demais tipos de alimento a oviposição foi significativamente menor e sempre muito baixa.

O maior nível de oviposição diária de N. anonymus foi observada quando o predador foi alimentado com T. mexicanus, atingindo dois ovos por fêmea; já com outro tetraniquídeo, $O$. gossypii, a oviposição diária foi pouco acima de um ovo por fêmea.

Estes níveis de oviposição foram maiores que aqueles obtidos por outros predadores quando alimentados com estes mesmos tetraniquídeos. Para os demais tipos de alimento, a oviposição foi menor que 0,5 ovo por fêmea, diferindo significativamente dos demais tratamentos. Esta espécie não ovipositou quando alimentada com T. heveae. 
Euseius concordis e A. floridanus apresentaram níveis de oviposição semelhantes quando alimentados com pólen. Polyphagotarsomnemus latus foi o alimento menos adequado para as espécies de predadores estudadas.

\section{DISCUSSÃO}

A aceitação de uma maior diversidade de alimentos por $A$. floridanus, indica que este ácaro possa se manter mais facilmente em seringueiras. Dentre as espécies estudadas, este pode ser o ácaro predador mais importante no controle de C. hevae e T. heveae, de vez que este foi o único predador a apresentar oviposição relativamente alta quando alimentada com aquelas espécies. Assim, em seringais onde se julgar necessário o controle químico de pragas, deve-se optar por produtos que não afetem a população de $A$. floridanus.

Trabalhando com Zetzellia mali Ewing, 1917, Delattre (1974) e SANTOS (1991) também constataram a preferência daquela espécie de estigmeídeo por eriofídeos em relação aos tetraniquídeos.

Os resultados deste trabalho são semelhantes àqueles obtidos por outros autores citados por MCMURTRY \& CROFT (1997), que também determinaram ser pólen o alimento preferido de espécies de Euseius Wainstein, 1962. Segundo McMurTRY \& Своғт (1997), espécies daquele gênero pertencem a um grupo de predadores que apresentam taxa reprodutiva mais alta quando alimentados com pólen, em comparação a outros tipos de alimento. Moraes \& Lima (1983) já haviam verificado ser pólen de Ricinus communis L., favorável à oviposição de $E$. concordis. Aqueles autores, além disso, obtiveram índices de oviposição relativamente altos quando E. concordis foi alimentado com o eriofídeo Aculops lycopersici (Massee, 1937), praga de tomateiro. Os resultados do presente trabalho indicam que nem todos os eriofídeos são aceitos por E. concordis como alimento, de vez que o nível de oviposição deste predador foi extremamente baixo quando $C$. heveae foi oferecido como alimento.

Poucos estudos têm sido conduzidos comparando-se o efeito de diferentes tipos de alimento na oviposição de $N$. anonymus, mas estes têm demonstrado a aceitação de tetraniquídeos como presa para aquele predador (Gondim et al. 1996, Mesa \& BellotTi 1986). No estudo conduzido por Gondim et al. (1996), N. anonymus apresentou oviposição diária de 1,5 ovos por fêmea, quando alimentado com T. urticae e Mononychellus tanajoa (Bondar, 1938), comparável aos resultados do presente trabalho. Segundo classificação estabelecida por McMurTRY \& Croft (1997), N. anonymus pertence a um grupo de predadores que apresentam alta taxa de oviposição alimentando-se de várias espécies de ácaros de Tetranychidae.

Fazolin \& Pereira (1989) relataram altas populações de tetraniquídeos em seringueira no Estado do Acre. Entretanto, de uma maneira geral, os ácaros desta família são encontrados em baixos níveis populacionais nesta cultura, o que possivelmente se dá pela ação dos ácaros predadores presentes na mesma. De acordo com os resultados observados neste estudo e pela freqüência observada por Ferla \& Moraes (2002), N. anonymus parece ser uma espécie importante no controle das populações de tetraniquídeos nesta cultura.

\section{AGRADECIMENTOS}

Às empresas Plantações Edouard Michellin Ltda. e Triângulo Agro-Industrial Ltda. pelo financiamento do projeto. Ao Conselho Nacional de Desenvolvimento Científico e Tecnológico $(\mathrm{CNPq})$ pela concessão de bolsa para a realização do doutorado do primeiro autor.

\section{REFERÊNCIAS BIBLIOGRÁFICAS}

Delattre, P. 1974. Étude de l'efficacité prédatrice de Zetzellia mali (Acarina: Stigmaeidae). Entomophaga, Paris, 19 (1): 13-31.

Fazolin, M. \& L.V. Pereira. 1989. Ocorrência de Oligonychus gossypii (Zacher, 1920) (Acari: Tetranichidae) em seringueiras cultivadas. Anais da Sociedade Entomológica do Brasil, Londrina, 18 (1): 199-202.

Feres, R.J.F. 1992. A new species of Calcarus Keifer (Acari, Eriophyidae, Phyllocoptinae) from Hevea brasiliensis Muell. Arg. (Euphorbiaceae) from Brazil. International Journal of Acarology, West Bloomfield, 18 (1): 61-65.

2000. Levantamento e observações naturalísticas da acarofauna (Acari, Arachnida) de seringueiras cultivadas (Hevea spp., Euphorbiaceae) no Brasil. Revista Brasileira de Zoologia, Curitiba, 17 (1): 157-173.

Ferla, N.J. \& G.J. de Moraes. 2002. Ácaros (Arácnida, Acari) da seringuiera (Hevea brasiliensis Muell. Arg.) no Estado do Mato Grosso, Brasil. Revista Brasileira de Zoologia, Curitiba, 19 (3): 867-888.

Flechtmann, C.H.W. \& R.J. Arleu. 1984. Oligonychus coffeae (Nietner, 1961), um ácaro tetraniquídeo da seringueira (Hevea brasiliensis) novo para o Brasil e observações sobre outros ácaros desta planta. Ecossistema, Espírito Santo do Pinhal, 9: $123-125$.

Gondim Jr., M.G.C.; G.J. de Moraes; J.V. Oliveira; R. Barros \& J.L.L. Pereira. 1996. Biologia de Neoseilus anonymus (Acari: Phytoseiidae). Anais da Sociedade Entomológica do Brasil, Londrina, 25 (3): 451-455.

McMurtry, J.A. \& G.T. Scriven. 1965. Insectary production of phytoseiid mites. Journal of Economic Entomology, College Park, 58 (2): 282-284.

McMurtry, J.A. \& B.A. Croft. 1997. Life-styles of phytoseiid mites and their roles in biological control. Annual Review of Entomology, Palo Alto, 42: 291-321.

Mesa, N.C. \& A.C. Bellotti. 1986. Ciclo de vida y hábitos alimenticios de Neoseiulus anonymus, predador de ácaros Tetranychidae en yuca. Revista Colombiana de Entomologia, Bogotá, 12 (1): 54-66.

Moraes, G.J de \& H.C. Lima. 1983. Biology of Euseius concordis (Chant) (Acarina: Phytoseiidae) a predator of the tomato russet mite. Acarologia, Paris, 24 (3): 251-255.

SANTOS, M.A. 1991. Searching behavior and association response of Zetzellia mali (Acarina: Stigmaidae). Experimental and Applied Acarology, Amsterdam, 11: 81-87.

Vieira, M.R. \& E.C. Gomes. 1999. Sintomas, desfolhamento e controle de Calcarus heveae Feres, 1992 (Acari: Eriophyidae) em seringueira (Hevea brasiliensis Muell. Arg.). Cultura Agronômica, Ilha Solteira, 8 (1): 53-71.

Recebido em 21.X.2002; aceito em 15.III.2003.

Revista Brasileira de Zoologia 20 (1): 153-155, março 2003 